Cinémas

Revue d'études cinématographiques

Journal of Film Studies

\title{
François, Damien, L'Immédiateté. Anthropologie culturelle critique, Berlin, Peter Lang, 2000, 441 p.
}

\section{Daniel Laforest}

Volume 12, numéro 1, automne 2001

Le Paysage au cinéma

URI : https://id.erudit.org/iderudit/024874ar

DOI : https://doi.org/10.7202/024874ar

Aller au sommaire du numéro

Éditeur(s)

Cinémas

ISSN

1181-6945 (imprimé)

1705-6500 (numérique)

Découvrir la revue

Citer ce compte rendu

Laforest, D. (2001). Compte rendu de [François, Damien, L'Immédiateté. Anthropologie culturelle critique, Berlin, Peter Lang, 2000, 441 p.] Cinémas, 12(1), 179-184. https://doi.org/10.7202/024874ar d'utilisation que vous pouvez consulter en ligne.

https://apropos.erudit.org/fr/usagers/politique-dutilisation/ 
FRANÇOIS, Damien, L'Immédiateté. Anthropologie culturelle critique, Berlin, Peter Lang, 2000, 441 p.

Il est ici question d'un livre curieux, d'un livre hors du commun. Son but avoué est celui d'esquisser les grandes lignes d'une anthropologie culturelle critique en lien avec l'un des problèmes intellectuels les plus accaparants de notre temps: la simultanéité dans la représentation et la perception du monde. Le livre de Damien François instaure une démarcation quant à l'ensemble des ouvrages pouvant de près ou de loin aborder la question, une démarcation marquée par la position initiale du regard critique. "L'anthropologie culturelle critique $[. .$.$] consiste en l'éva-$ luation de tout phénomène, du plus intellectuel au plus trivial, qui participe de la présence de l'homme dans le monde et des conséquences de cette présence" (p. XIII). Limpression d'originalité que procure la démarche se trouve ainsi amorcée dans l'apparente indifférence quant à la nature du matériau culturel glané par les recherches (à l'arrivée, essentiellement des extraits d'ouvrages à vocation scientifique, des extraits de romans populaires ou plus élitistes, des paroles de chansons pop ou rock, des références au cinéma avec extraits de scénarios à l'appui, aux arts plastiques et à la bande dessinée, de même que des échantillons de publicités). Une telle entreprise, s'affublant du nom d'anthropologie, peut attirer la réprobation immédiate d'une certaine critique qui, en pointant son évidente subjectivité, passera outre avec dédain. L'auteur tente, dans l'ouverture de son ouvrage, de parer les éventuels coups qu'on lui assenera à travers des paroles qui révèlent à la fois l'ambition et la portée souhaitée de ses réflexions: «... je suis convaincu qu'il s'agit là au contraire de la seule méthode de travail qui soit en mesure de rendre compte du cheminement de ma pensée, de la pensée critique 
tout court. Cette coexistence de moments subjectifs ne garantitelle pas l'objectivité, MON objectivité?"(p. XIV). Une telle fronde ne peut que retenir l'attention; et le livre pourra révéler une cohérence insoupçonnée, dans la constitution même de son message, à qui voudra bien contourner l'habituel dédain superficiellement entretenu face à la notion de subjectivité en sciences humaines.

Construire un discours significatif articulé autour de la simultanéité (et plus spécifiquement autour du sentiment qu'elle procure: l'immédiateté) peut s'avérer une occasion de subir un cuisant naufrage méthodologique et analytique. Le sujet est infini et surtout irréductible puisqu'il touche le fonctionnement même de l'appréhension du monde par la pensée. Damien François en est bien sûr conscient et a construit sa réflexion en conséquence. Situé, par le biais des compétences particulières de l'auteur, au confluent des études cinématographiques et filmiques, de la narratologie, des théories de la communication et de la philosophie, L'Immédiateté se présente dans son essence comme l'analyse d'un roman, Le Sursis, publié en 1945 par Jean-Paul Sartre. Pourquoi donc ce choix d'un penseur certes immense, mais auteur d'une littérature tellement ancrée dans sa modernité que peu se hasardent à en faire l'assise épistémologique de leurs réflexions sur notre époque contemporaine? Pourquoi aussi choisir ce roman mal connu, grandement dévalué depuis sa publication en raison d'une construction jugée laborieuse, fatigante pour le lecteur car axée sur une juxtaposition non linéaire d'événements diégétiques sans rapports apparents entre eux? La réponse, tente de nous dire Damien François, se situerait dans une incompréhension initiale, une triste méprise sur les intentions philosophiques que sous-tend la construction narrative du roman. On aurait occulté le formidable caractère visionnaire de cette cuvre simplement parce qu'on l'aurait jugée trop peu littéraire (ou alors trop peu efficace d'un point de vue littéraire). Et pourtant il semble que c'est ce qui ferait justement toute sa force.

Dans son ouvrage, Damien François vise à démontrer que la narration et la discursivité du Sursis ne peuvent révéler leur plein potentiel qu'à travers la théorie générale du montage cinématographique. En d'autres termes, Sartre aurait en toute conscience 
construit un roman cinématographique. Sa technique de narration serait proche du montage en ce sens qu'elle lui emprunterait la liberté d'accoler les phrases, de même que les différents types de focalisation, sans l'aide par trop littéraire des indicateurs spatiaux et temporels, ou des marqueurs de relations classiques. Le but de Sartre, celui-là même qui en fait selon François un précurseur parfait de nos conceptions actuelles de la simultanéité, aurait été de représenter une réalité globale (l'aube de la guerre en Europe), partagée par toutes les subjectivités, dont même la plus exhaustive somme des causalités et des effets observables ne peut approcher la vérité. Le Sursis serait un roman de la transcendance recherchée, quoi qu'on en dise, par la philosophie sartrienne. Une transcendance exprimée dans la contamination de toutes les consciences par une réalité englobante, mais impensable par une seule d'entre elles, car se heurtant toujours à la barrière de la subjectivité. Ainsi François en arrive à affirmer que «... la polysémie née du montage des segments engendre la concrétisation philosophique de l'universel singulier» (p. 299), ce concept - l'universel singulier — étant donc la concrétisation de la transcendance chez Sartre. Selon François, qui se réclame en ce sens d'Eisenstein, le montage aura été non pas simplement la construction d'un nouveau langage à travers le matériau filmique, mais aussi une révolution sans précédent dans notre perception du monde (p. 379):

Avec les progrès fulgurants des sciences cognitives, on pourrait aujourd'hui envisager une théorie d'ensemble du rnontage en tant que système fondateur de notre perception, représentation et compréhension de tout univers - diégèse "fictionnelle " ou monde "réel " qui, formellement, se ressemblent toujours [...].

Mais qu'est-ce donc que l'immédiateté à travers cette perspective, sinon la pensée s'essayant à l'impossible contemplation de son propre fonctionnement? En effet perception, appréhension et compréhension ne se décomposent jamais pour s'offrir ainsi à notre esprit. Leur enchaînement est lui-même impossible à représenter autrement que par la médiation qui permet de dompter les contraintes que sont l'espace et le temps, ces 
contraintes que la pensée pure ignore. C'est donc la médiation qu'il faudra toujours penser, et celle-ci ne sera plus qu'affaire de vitesse dans un perpétuel élan vers l'immédiateté. Damien François perçoit l'organisation communicationnelle de notre temps comme une cristallisation de cette aspiration qu'il soupçonne avoir toujours existé dans la psyché humaine (p. 328):

\section{[...] en effet, il faut peut-être chercher le paradigme distinctif des "nouveaux médias" uniquement du côté de leur vitesse d'exécution. [...] L'homme moderne semblerait par conséquent avoir trouvé, en la vitesse, un moyen infaillible d'apaiser sa soif d'immédiateté, c'est-à-dire de couper court à toute médiation [...].}

Voilà exposé, bien que de manière très schématique', le propos général de L'Immédiateté. Cependant la valeur novatrice de l'ouvrage de Damien François ne se situe pas exactement à ce niveau. Il faudra la chercher dans une citation de Theodor Adorno, deux fois répétée, en forme de leitmotiv (p. 141): "Tout sens d'un texte littéraire est véhiculé par sa forme. " Force est de reconnaître que la forme donnée à L'Immédiateté dépasse en singularité tout ce qu'on peut habituellement observer dans la sphère étendue des ouvrages universitaires. L'anthropologie culturelle critique, rappelons-le, se veut une discipline amenant l'éventuel chercheur à manipuler une foule de "données", sans droit de regard sur l'importance esthétique ou hégémonique de celles-ci dans la culture choisie ${ }^{2}$. La façon dont François intègre son corpus de recherche à la narration de type essayiste, qui fait le corps de l'ouvrage, présente un visage inédit de la démarche critique, même au sein des cultural studies, desquelles on peut vaguement rapprocher L'Immédiateté.

L'ouvrage de Damien François est composite. Ceci non pas au sens structuraliste où l'auteur canaliserait inconsciemment un faisceau de savoirs préexistants, mais bien de manière tout à fait manifeste, conceptuelle même. Il s'agit bien d'intertextualité, mais d'une intertextualité revendiquée au point d'être érigée en véritable esthétique. Le discours n'appuie pas ici son propos sur un nombre restreint de citations, qu'on aurait choisies consciencieusement afin d'offrir une progression aisée et quelque peu 
scolaire dans l'argument. Bien au contraire. On pourrait dire qu'il s'entremêle à un tissu d'extraits de textes parfaitement diversifiés, exactement comme la parole s'ajoute au monde non pas en s'imprimant sur sa surface, mais en s'immiscant dans son corps comme en un nœud où s'entrecroiseraient toutes les autres paroles. Très souvent, par exemple, une phrase emprunte une minime portion d'un autre texte, voire les paroles d'une chanson populaire, afin de constituer son complément. Ou alors le lecteur tombe sur un passage important du discours, amorcé par l'instance narrative (le fameux "Je" de l'essai) et complété par les paroles d'un autre auteur, parfois le plus inattendu comme dans ce glissement presque imperceptible qui s'opère vers une citation de Maurice Blanchot (François, p. 379):

Lentreprise sartrienne du Sursis transforme elle aussi le lecteur en conscience parallèle du monde et thématise, à côté des thèmes "évidents" que sont la genèse de la Seconde Guerre mondiale et la simultanéité, le "savoir instantané, universel, [qui] fait de nous le pur passage d'un mouvement où chacun s'est toujours déjà, par avance, échangé contre tous " ${ }^{3}$.

À bien des égards, la discursivité de François s'exprime par la citation ${ }^{4}$. C'est ce qui nous permet de soupçonner que la forme donnée par son auteur à L'Immédiateté contient en elle-même une grande part de son propos, si ce n'est l'image complète de celui-ci. Le lecteur ne sera pas surpris, suivant ceci, de lire ces lignes dans la "déclaration d'intention" qui ouvre le livre (p. XV) :

Je n'ai nullement cherché à briller par un encyclopédisme abusif, j'ai simplement voulu "mettre sur la table " la généalogie de ma pensée. [...] Oui, tout est lié, quu'il s'agisse de langage cinématographique, de psychologie cognitive ou de publicité mensongère. Mon rôle se résume ici finalement à celui de monteur [...].

Le montage, tel que l'entend François, consiste en ce langage qui se calque sur le fonctionnement de la pensée afin de supprimer les intermédiaires causals entre les segments du discours, de manière à laisser l'imagination combler les espaces de raccords 
où doit se faire le lien. Si "tout est lié", l'essayiste qui veut offrir une représentation opérante du réel, c'est-à-dire conforme à la façon dont celui-ci s'appréhende aujourd'hui, devra reconnaître l'importance et le caractère simultané de ces liaisons. Au-delà des évidents problèmes et embourbements stylistiques qu'implique la structure textuelle de L'Immédiateté, c'est dans cette reconnaissance et dans la louable tentative d'exprimer celle-ci formellement que le livre de François s'avère unique et séduisant.

\title{
Daniel Laforest
}

\author{
Université du Québec à Montréal
}

\section{NOTES}

1. Il convient d'indiquer que l'auteur offre une analyse narratologique très rigoureuse du roman Le Sursis. D'autre part, une grande partie de son livre est construite selon une méthode d'allées et venues, nous ramenant sans cesse au cour du propos, qui n'est pas simplement de montrer l'originalité du roman de Sartre, mais aussi de tisser des liens entre celui-ci et les actualisations de l'immédiateté dans la culture contemporaine.

2. On est en droit de se demander, toutefois, quelle est la culture précise sur laquelle porte la réflexion de François. Le contexte de la mondialisation économique et médiatique permet-il à lui seul de parler sans ambages d'une "culture occidentale" aux contours grossiers, informes et vaguement américanisés?

3. La deuxième partie de la citation appartient à Maurice Blanchot (1959, p. 246).

4. Ceci donnant lieu à une débauche inouie de notes de bas de page, qui doivent être considérées comme appartenant au discours au même titre que le texte principal puisqu'elles commandent, à travers l'importance des digressions qu'elles comportent, une lecture qui doit passer par elles.

\section{RÉFÉRENCES BIBLIOGRAPHIQUES}

Blanchot, 1959 : Maurice Blanchot, Le Livre à venir, Paris, Gallimard, 1959. 